\title{
La géopolitique pour comprendre le contexte socio-culturel libanais et ses pratiques linguistiques
}

\author{
Ludivynn MUNOZ \\ Université Paris 1
}

\section{Résumé}

Notre problématique concerne l'intérêt d'une démarche géopolitique en vue d'éclairer des contextes plurilingues et multiculturels. Le but est de montrer les apports que pourrait soutenir cette discipline dans la contextualisation didactique, en vue d'adopter les meilleures décisions. Quelles sont les caractéristiques de cette discipline qui ré-émerge depuis quelques années? Et comment la géopolitique peut aider à mieux cerner les contextes complexes de certains territoires ? Le cas du Liban est étudié pour mettre en exergue les contributions de cette discipline dans ce contexte socio-culturel complexe, à savoir des profils d'usage de la langue française distincts spontanés ou stratégiquement mis en œuvre.

\section{Mots-clés}

Liban, Francophonie, géopolitique, langues.

\begin{abstract}
Our problem concerns the interests of a geopolitical approach to explain multilingual and multicultural contexts. The aim is to show the contributions that could support this discipline in contextualizing teaching to adopt the best decisions. What are the characteristics of this discipline which re-emerge in recent years? And how geopolitics can help to better understand the complex contexts of certain territories? The case of Lebanon will be designed to highlight the contributions of this discipline in this socio-cultural complex.
\end{abstract}

\section{Keywords}

Lebanon, Francophonie, geopolitics, language. 


\section{Introduction}

L'objectif est de mettre en avant l'intérêt d'une démarche géopolitique dans l'analyse d'un contexte plurilingue et multiculturel, puisqu'elle apparait importante pour éclairer les processus de contextualisation didactique. Pour avancer des éléments en ce sens, nous prendrons en compte le cas du Liban, en ce qu'il cristallise des représentations linguistiques potentiellement conflictuelles. Le Liban est un pays méditerranéen arabe, peuplé de 4,29 millions d'habitants (dont 1,5 millions dans l'agglomération de Beyrouth). Ce pays connaît une importante diaspora dans le monde entier (avec 15 millions de personnes hors des frontières libanaises); ce qui en fait à notre connaissance l'un des rares pays au monde dont la population extérieure est trois fois plus importante que la population interne. En outre, le Liban qualifié d'ancienne Phénicie, est réputé pour un être un pays commerçant depuis ses origines. Ces deux états de fait nous amène à fonder l'hypothèse que le Liban serait plus prompte à s'ouvrir sur le monde, favorisant ainsi le multilinguisme dans ce pays.

Notre étude se base sur une enquête réalisée à Beyrouth, de mars à juin 2010, à partir d'entretiens menés auprès d'une cinquantaine de personnes, essentiellement acteurs d'instances relevant plutôt de la sphère de socialisation secondaire (professeurs, personnels d'agence de promotion des langues, journalistes, personnels et dirigeants politiques) afin de considérer les processus de socialisation dépassant les processus de socialisation de type primaire (essentiellement construits dans le cadre des familles) que propose Berger et Luckmann $(1966)^{1}$. Notre canevas d'entretien s'organisait autour de trois thèmes relatifs aux pratiques linguistiques des personnes interrogées en famille et au travail, leurs représentations des langues en présence sur le territoire libanais, ainsi que sur le plurilinguisme et ses conséquences. Cette enquête a permis de recueillir des données avant tout qualitatives relatives aux représentations des langues présentes et utilisées sur le territoire libanais, et dans une moindre mesure, des données quantitatives sur la fréquence d'utilisation des différentes langues en présence. Nous examinerons plusieurs ordres de questions qui viseront à éclairer ce contexte selon les deux formes de socialisation primaire et secondaire. Pourquoi les parents choisissent de scolariser leurs enfants dans des écoles francophones ? Qui utilise la langue française au Liban au quotidien? La question du statut du français sera également évoquée : est-il une langue seconde au Liban ou une langue étrangère ? Le français est-il présent dans les médias libanais ? Ces questions relèvent de la géopolitique des langues, mais le problème central que nous soulevons est de savoir comment cette discipline, qui ré-émerge depuis quelques années, peut-elle aider à mieux cerner les contextes complexes de certains territoires comme le Liban.

Notre propos se déploiera en trois mouvements. Dans un premier mouvement nous montrerons les atouts de la démarche géopolitique, notamment celle ayant trait à la géopolitique de la langue française (Lacoste, 2003 ; Giblin, 2007) en ce qu'elle situe au cœur de son raisonnement les rapports de force et les représentations des langues (Breton, 2003, 2006). Dans un deuxième mouvement nous découvrirons pourquoi la langue française est présente sur le territoire libanais, en nous centrant sur l'héritage historico-culturel de ce pays. Au sein d'un troisième mouvement, nous nous centrerons sur le contexte socioculturel et identitaire libanais, où se côtoient actuellement l'arabe, le français et l'anglais. L'apparition de l'anglais concurrence la langue française, jusqu'alors majoritaire avec l'arabe dans les processus d'apprentissage (Fitouri, 1983).

\footnotetext{
${ }^{1}$ Parmi la synthèse des théories liées aux processus de socialisation établies par Dubar (1991), ce dernier distingue celles qui se caractérisent par une distinction primaire/secondaire liée à l'âge de la personne de celles distinguant ses réseaux de sociabilité. C'est cette dernière que nous adoptons.
} 


\section{Pour une géopolitique des langues}

\subsection{La géopolitique}

La géopolitique est la discipline qui étudie les rapports de force pour des territoires que se livrent des groupes d'acteurs dont les représentations ayant trait au territoire divergent. Par exemple, les Flamands n'ont pas la même vision de la Belgique que les Wallons, même si aucun rapprochement hâtif et amalgame ne doivent s'opérer².

Selon Giblin (2007) : « l'approche géopolitique met l'accent sur les relations de pouvoir au sein des sociétés et sur les représentations et convictions défendues par les individus et les groupes. Elle prend en compte à la fois la culture et la politique afin de jauger, (...) la place de l'une et l'autre ainsi que leurs interactions ". De ce fait, cette discipline se confronte au sens communément construit par les acteurs et les institutions. En effet, la culture et les langues peuvent influer sur le politique, car «les langues constituent d'importants enjeux géopolitiques » selon Lacoste (2003). De surcroît, des groupes peuvent les mobiliser pour défendre leurs causes, leur place sociale dans la société, par exemple. La langue peut être au cœur de nombreux enjeux politiques dans un pays ou sur un territoire. Très souvent, des groupes indépendantistes (comme au Pays Basque ou encore au Québec) vont mettre en avant une langue et une culture spécifique; la langue peut même devenir fondamentale dans les revendications affichées.

Les personnes au pouvoir tentent d'imposer leurs cultures ou très souvent la culture du groupe dominant, le plus souvent celle des élites, et c'est ainsi que de nombreuses cultures ou langues sont minorées pour des raisons politiques. Certains des pays n'hésitent pas à reconnaître des minorités culturelles et/ou linguistiques pour les protéger, mais pour d'autres, ceci apparaît plus difficile; en témoigne la réticence de nombreux pays européens pour signer la Charte européenne des langues régionales ou minoritaires (Plasseraud, 2005).

Lorsqu'un pays possède une ou plusieurs langues officielles, les locuteurs des différentes langues peuvent essayer d'imposer la pratique de leurs langues respectives; raison pour laquelle des États vont mettre en place des politiques de quotas au sein de leurs personnels d'États, où un certain pourcentage de personnes parlant telle langue ou issu de telle minorité doit être embauché. De nombreux pays bilingues (Canada, Belgique), pour une meilleure égalité entre les langues et les communautés, instaurent des quotas ou des lois de protection pour réglementer leurs usages : par exemple imposer l'affichage dans une ou plusieurs langues pour les panneaux commerciaux ou publics.

En Belgique, trois communautés et trois régions furent reconnues : la communauté flamande formant la région flamande, la communauté française et la communauté germanophone formant la région wallonne, la troisième région est formée par le statut particulier de la capitale avec la région de Bruxelles-capitale (Giblin, 2010) . $^{3}$

\footnotetext{
${ }^{2}$ Il suffit de voir que la Belgique peut mettre de nombreux mois pour constituer un gouvernement en raison des différents qui divisent les Wallons et les Flamands; et le cas du statut de Bruxelles-Capitale est une illustration de cette vision divergente entre Wallons et Flamands (les Flamands considérant que Bruxelles se trouvent en territoire flamand alors que les Wallons pensent que Bruxelles est un territoire francophone puisque la capitale est peuplée par une majorité de francophones) (De Montbrial et Jansen, 2007 ; Giblin, 2010).

${ }^{3} \mathrm{http} / /$ www.belgium.be/fr/la_belgique/pouvoirs_publics/la_belgique_federale/Carte/
} 
Au Canada, c'est seulement à partir de la fin des années 1960 que l'association territoriale entre les Canadiens français et le Québec commence à se réaliser (ceci fait partie de « la révolution tranquille »). La loi $101^{4}$ fut instaurée au Québec pour protéger la langue française menacée de disparition. Au Canada, tout le monde (du ministre aux gardes de douane) est censé être bilingue ainsi que toutes les productions administratives et tous les produits manufacturés (la réalité est souvent toute autre), mais ceci favorise les francophones dans l'accès aux emplois administratifs, car ils ont plus tendance à être bilingues du fait du rôle minoritaire que joue la langue française au Canada. Tout doit être en français comme en anglais, c'est pour cela qu'on subventionne des radios en français par exemple.

La géopolitique met au centre de son étude les représentations des langues en présence. Il s'agit dès lors de savoir si la langue est appréciée ou non, et auprès de quel public ? En effet, les individus et les groupes (liés aux différents types d'acteurs) peuvent mobiliser une langue pour l'étendre sur ce qu'ils considèrent comme relevant de leur territoire légitime. Il peut donc se mettre en place un rapport de force entre les langues, et entre les groupes qui les mobilisent et les utilisent. A cet égard, notre principale question se reformule de la manière suivante. Existet-il au Liban un groupe d'acteurs qui défend l'usage d'une langue particulière ?

De plus, la politique internationale joue aussi un certain rôle. Suite à l'opposition de la France à la guerre en Irak en 2003 (et donc à la non présence de soldats Français sur le territoire irakien), cette dernière a bénéficié d'une image positive dans la région Moyen-Orient ; ce qui peut pousser des personnes à apprendre le français, même si ceci ne dure qu'un temps comme l'a précisé l'attachée de coopération pour le français au SCAC (Service de Coopération à l'Action Culturelle) de l'Ambassade de France à Beyrouth.

\subsection{Le pouvoir des langues}

Dans son ouvrage intitulé Horizons et frontières de l'esprit, comprendre le multiculturalisme, Roland Breton (2006) évoque cinq systèmes d'enfermements. Ces systèmes peuvent, au contraire, également apparaître comme des facteurs d'ouverture lorsqu'il y a communauté et partage de ces entités. L'auteur les classe par ordre décroissant (en fonction de leur impact) : «la langue, premier système d'enfermement, l'ethnie ou la fermeture sociale, la culture, fermeture mentale, les groupes et appareils, fermetures sociales, le territoire de l'État ${ }^{5}$ : fermeture géopolitique $»$. Il existe donc une fermeture sociale locale ethnique et des fermetures sociales plus "globales ». Ainsi indique-t-il en premier lieu de son système de catégorisation, la langue. Des groupes d'acteurs peuvent donc être «enfermés » au sein de leur communauté linguistique; ils auront tendance à protéger leur langue commune et peut-être même à l'imposer sur leur territoire. Territoire et langues sont liés et la géopolitique étudie les rapports de force entre différents groupes d'acteurs pour un même territoire. La langue peut apparaître comme un outil politique pour ces groupes d'acteurs, nécessaire mais toutefois non suffisant. Le linguiste Claude Hagège (2008) explique pourquoi les langues s'imposent :

«Comment certaines langues sont-elles imposées ? Toute langue en a les moyens, mais toutes ne bénéficient pas (...) d'une promotion politique ou rhétorique. (...) Mais l'autorité politique ne suffirait pas à faire de la langue un instrument de pouvoir, sans le poids démographique de ceux qui la parlent. La démographie est en effet, en ce domaine, l'auxiliaire de l'entreprise politique ».

\footnotetext{
${ }^{4}$ Loi 101 du gouvernement québécois : http://www2.publicationsduquebec.gouv.qc.ca/dynamicSearch/telecharge.php?type=2\&file=/C_11/C11.html ${ }^{5}$ C'est nous qui soulignons par l'usage de l'italique
} 
Ce qui va permettre à une langue de s'imposer sur un territoire c'est son statut politique, si les élites politiques la mobilisent et officialisent ses usages, mais, et/ou par voie de conséquence, également son nombre de locuteurs (permettant d'en déduire le théorème suivant: plus une langue a de locuteurs plus elle aura tendance à dominer).

L'approche géopolitique devrait nous permettre de mieux comprendre le plurilinguisme actuel du Liban, qui consiste en une situation relativement complexe, puisque enchevêtrée dans de multiples territoires et de multiples contextes, qu'il s'agit d'éclairer.

\section{Le français au Liban}

\subsection{Présence et statut du français au Liban}

Le français est présent au Liban pour des raisons historiques. En effet, au sortir de la première guerre mondiale, le Royaume-Uni et la France obtiennent des mandats de la Société des Nations (SDN, ancêtre de l'Organisation des Nations Unies) sur d'anciens territoires de l'Empire Ottoman : Irak et Palestine pour le Royaume-Uni et Grand Syrie pour la France (Khoury, 2006 ; Faour, Velut et Verdeil, 2007). Ce sont les accords de Sykes-Picot en 1916 qui vont répartir les zones d'influences françaises et britanniques dans la région du Proche-Orient. La France obtient un mandat sur la Syrie dont le Liban était une entité administrative. C'est grâce à la puissance mandataire française que l'État libanais a pu se constituer. Depuis l'indépendance en 1943, le pays a connu une guerre civile à partir de 1975 qui s'est terminée par les accords de Taef en 1990 durant lesquels la France n'était pas présente.

Le territoire libanais fut sous mandat de la France de 1920 à 1943. Le Grand Liban fut crée le $1^{\text {er }}$ septembre 1920 par le général Gouraud, pour protéger la communauté chrétienne maronite, à la Résidence des Pins à Beyrouth et ainsi le différencier de la Grande Syrie.

Le pays fut indépendant le 22 novembre 1943. Au sortir de l'indépendance, le sort du français n'a pas réellement été fixé. Dans la constitution de 1926, révisée par la loi constitutionnelle du 9 novembre 1943, il est stipulé dans l'article 11: «L'arabe est la langue nationale officielle dans toutes les administrations d'État. Une loi spéciale déterminera les cas où il sera fait usage de la langue française ${ }^{6}$. L'arabe est la langue officielle de l'État libanais, et les cas d'usage de la langue française n'ont jamais été définis dans une loi spéciale et ne le seront sûrement pas, puisque la politique intérieure libanaise est déjà assez riche en événements pour que les politiques se préoccupent du statut des langues en présence sur le territoire libanais. Même après les accords de Taëf, en 1991, qui ont vu la constitution modifiée, cela n'a pas changé. Le statut et le rôle du français au Liban sont ambigus puisque rien ne précise réellement ce qui est obligatoire et ce qui ne l'est pas. L'administration et les ministères acceptent les documents en français. Depuis 1992, tout représentant de l'État libanais doit s'exprimer en arabe sur le territoire libanais, et il lui est désormais possible de le faire en français ou en anglais à

\footnotetext{
${ }^{6}$ Il existe deux versions différentes de cet article 11. Rien n'est réellement clair et tout peut être sujet à polémique, la majorité des versions trouvées propose le texte cité ci-dessus : «L'arabe est la langue nationale officielle dans toutes les administrations d'État. Une loi spéciale déterminera les cas où il sera fait usage de la langue française ». Dans une autre version ancienne, il est possible de découvrir que le français serait également langue officielle : « L'arabe est la langue nationale officielle dans toutes les administrations d'Etat. Le français est également langue officielle, une loi spéciale déterminera les cas où il en sera fait usage » (http://conseilconstitutionnelliban.com/subfr.aspx?id=169, disponible le 28 juillet 2011, Site du Conseil Constitutionnel libanais). Le conseil constitutionnel libanais publie les deux versions en précisant que celle où le français est langue officielle est la version ancienne, alors que l'autre version est celle qui a été modifiée par la loi constitutionnelle du 9 novembre 1943. En 1943, à l'indépendance, le statut de langue officielle pour le français a été annulé.
} 
l'étranger. Au niveau juridique, le français a une certaine importance, puisque le droit libanais est très proche du droit français : de nombreuses lois libanaises ont été rédigées en français durant le mandat et sont encore valables; la jurisprudence est largement inspirée des codes et d'ouvrages français.

Dans la vie de tous les jours au Liban, il est possible de voir qu'il est réservé au français certains usages officiels : toutes les plaques minéralogiques sont en français et en arabe, ainsi que les billets de banques avec une face en français et une face en arabe.

Il faut préciser que le Liban ne possède pas de réelles statistiques, et le dernier recensement date de 1932 (sous le mandat français). Tout recensement apparait impossible dans le but de protéger les différents antagonismes qui pourraient naître entre les différentes communautés (sièges au parlement, poste ministériels, etc.). Le nombre de francophone est estimé à 1,6 millions ( $38 \%$ de la population en 2003). Comme aucune statistique fiable n'est disponible (car «aucun recensement de la population n'a été effectué afin de ne pas officialiser l'inégale croissance démographique de communautés plus ou moins rivales » selon Lacoste, 2009), la population réellement francophone pourrait être de 50\%, mais rien n'est assuré.

Au Liban, le français est à la fois langue d'enseignement (notamment dans les matières scientifiques et techniques), langue seconde et langue étrangère au même titre que l'anglais. Mais il a également la particularité d'être quelquefois langue maternelle, puisque des parents l'utilisent avec leurs enfants pour faciliter par la suite leur insertion à l'école (plusieurs parents libanais interrogés m'ont précisé opter pour cette stratégie). Ce qui semble pertinent puisqu'en effet, sur 900000 élèves Libanais, 600000 sont scolarisés dans un établissement francophone.

\subsection{Les représentations sur le français}

Au Liban, le français a été associé à la communauté chrétienne ${ }^{7}$ et plus précisément aux maronites car pendant le mandat « les autorités françaises s'appuyèrent sur une partie de la population, les chrétiens maronites d'obédience catholique » (Lacoste, 2009) et il est également lié aux classes sociales aisées.

L'enquête a été réalisée à Beyrouth, de mars à juin 2010, auprès de 49 personnes, acteurs d'instances de socialisation secondaire agissant au sein d'institutions susceptibles d'impacter, au delà des processus de socialisation primaire liée à l'activité des familles, sur les représentations des enfants et des adolescents en développement (cf. Tableau 1). Il s'agissait plus précisément d'entretiens semi-directifs menés à partir d'un canevas d'entretien relatif aux pratiques des langues en présence sur le territoire libanais, leurs représentations, leurs fréquences et leurs modes d'usages.

\footnotetext{
7 «Les chrétiens libanais (divisés en 12 églises, dont les maronites, francophones et les grecs orthodoxes anglophones) » in Lacoste, Y. (2009). Géopolitique, la longue histoire d'aujourd'hui. Paris : Larousse.
} 


\begin{tabular}{|l|c|}
\hline \multicolumn{1}{|c|}{ Catégories d'acteurs } & Nombre \\
\hline Responsables et représentants francophones au Liban & 3 \\
\hline Employés du Bureau Moyen-Orient de l'Agence Universitaire de la Francophonie & 15 \\
\hline Responsables des centres culturels européens & 4 \\
\hline $\begin{array}{l}\text { Responsables de Départements Universitaires de Français (DUF) en 2010 dans des } \\
\text { universités du Moyen-Orient }\end{array}$ & 7 \\
\hline Employés d'ambassades et représentations d'organisations supranationales & 5 \\
\hline Boursiers et ex-boursiers de l'Agence Universitaire de la Francophonie & 8 \\
\hline Responsables de médias libanais Total & 7 \\
\hline \multicolumn{1}{|c|}{$\quad$ 49 } \\
\hline
\end{tabular}

\section{Tableau 1 : Répartition de l'échantillon d'enquête}

L'étude des représentations sur les langues présentes sur le territoire libanais fut réalisée à partir de questions posées à l'échantillon. Nous présenterons quelques unes de ces questions et quelques résultats dans cette sous partie.

Voici un exemple de résultats relatif aux représentations quant à l'usage des langues au Liban (cf. Tableau 2).

\begin{tabular}{|l|c|c|c|}
\hline & Arabe & Français & Anglais \\
\hline Langue culturelle & 43 & 41 & 14 \\
\hline Langue commerciale & 13 & 12 & 43 \\
\hline Langue diplomatique & 13 & 25 & 21 \\
\hline Langue politique & 20 & 19 & 25 \\
\hline
\end{tabular}

Tableau 2 : Répartition des réponses des personnes enquêtées

D'un premier abord, il est notable d'avoir à constater que les fonctions linguistiques des trois langues semblent très variées quant à leur répartition, sauf pour la seconde, très tranchée, puisqu'elle se dégage nettement des trois autres, et encore plus de la dernière, où les résultats ne devraient pas être significativement différenciateurs.

En ce qui concerne chacune des langues, il est possible de retenir les principaux éléments suivants.

Du côté de l'arabe, pour la majorité des personnes rencontrées, elle apparaît comme une langue culturelle. Moins de la moitié la considère comme une langue politique alors que celle-ci est souvent la première, si ce n'est la seule, langue utilisée dans les instances politiques libanaises. Enfin, celle-ci n'est pas réellement considérée comme une langue commerciale ou diplomatique.

Du côté de la langue française, tout comme l'arabe, elle est majoritairement considérée comme une langue de culture. Plus de la moitié des personnes interrogées la mentionne en tant une langue diplomatique. La représentation et l'héritage du français comme langue diplomatique semblent par conséquent encore prépondérants; ce qui laisse largement à penser qu'à ce niveau, l'anglais n'aurait pas, pour l'instant, pris totalement le dessus. Un peu moins de la 
moitié opte pour le français en tant que langue politique. Seulement un quart perçoit le français comme une langue de commerce, alors même que de grandes entreprises françaises sont implantées au Liban, appartenant à divers secteurs (comme le secteur agro-alimentaire avec Candia, Danone, etc. $)^{8}$. En ce qui concerne ce point particulier, une hypothèse serait relative au fait de savoir quelle est la langue d'usage (du français ou de l'anglais) de ces multinationales françaises au Liban. Peut-être vont-elles bien préférer traiter leurs affaires en anglais même si l'usage du français sera possible?

Concernant l'anglais, cette langue est très majoritairement perçue comme une langue de commerce. Plus de la moitié des personnes interrogées la voit comme une langue politique. Un peu moins de la moitié pense qu'il s'agit d'une langue diplomatique. Et seulement guère plus d'un tiers, la qualifie de langue culturelle.

Grâce à deux questions posées à l'échantillon (Question 10: Dans quel contexte, milieu et territoires utilisez-vous la langue française ?; Question 12: A quels pays et quelles valeurs associez-vous la langue française ?), il est possible de se rendre compte que le français est une langue fortement connotée au Liban; elle n'est pas neutre et est chargée de moult représentations. Dans ces deux questions posées à notre échantillon, qui n'évoquaient pas directement l'affiliation entre chrétiens et francophones, certaines personnes ont cité ce rapprochement et cette représentation. A ce titre, onze pensent que les francophones sont chrétiens : une précisait que «les chrétiens maronites sont plus francophones mais les chiites libanais en Afrique francophone le sont également ». Le facteur linguistique n'est pas forcément un facteur conflictuel au Liban. Le français est lié aux chrétiens et plus précisément aux maronites, mais il est également lié aux classes sociales aisées et apparaît comme une langue élitiste (le terme de « langue de salon » est utilisé par les Libanais mais cette représentation ne touche pas uniquement le Liban, et apparaît également dans d'autres pays, historiquement certainement en raison de son usage diplomatique ou encore dans certaines cours royales européennes comme celle de Russie - Rjeoutski, 2007) au contraire de l'anglais qui, grâce à son atout de langue commerciale, pourrait permettre à tout le monde de «faire fortune ». Le français est une langue vivante au Liban: même si son rôle est surtout développé dans le système éducatif, elle est également utilisée pour la vie quotidienne et certains Libanais l'utilisent plus que l'arabe. A la Question 11 «selon quelle fréquence utilisez-vous le français ? Tous les jours - Tous les mois - Toutes les semaines - Très rarement », 43 personnes interrogées ont répondu « Tous les jours ».

Ces entretiens et les réponses aux questions ont permis de recueillir bon nombre d'informations et de percevoir des représentations fortement ancrées au Liban. Il est également possible de tirer quelques conclusions à partir de l'analyse des données. Plus généralement les différentes langues en présence sur le territoire libanais sont en confrontation directe les unes avec les autres. La langue arabe tient une place centrale en tant que langue véhiculaire et officielle du Liban, mais elle peut être concurrencée par le français et l'anglais. Cependant, le plurilinguisme reste fondamental au Liban, car les institutions le reconnaissent. L'arabe constitue la langue véhiculaire au Liban, il est la langue maternelle de la plupart des Libanais. Le français est une langue prépondérante dans le système éducatif libanais, c'est aussi la langue maternelle de quelques Libanais qui la préfèrent à l'arabe. Comme nous l'avons indiqué, elle est cependant très fortement associée à la communauté chrétienne (plus particulièrement aux maronites) et

\footnotetext{
${ }^{8}$ La France était en 2009 au premier rang de l'Union Européenne (918 milliards d'euros) et au troisième rang mondiale pour les Investissements Directs à l'Etrangers (IDE) (1191 milliards de dollars étatsuniens) selon la revue "Quai d'Orsay, Une diplomatie en danger?», Diplomatie, Affaires Stratégiques et Relations Internationales, septembre - octobre 2010, numéro 46, Areion, 90 pages.
} 
aux classes sociales aisées. L'anglais prend de plus en plus de place au Liban et commence, semble-t-il, à concurrencer le français dans le système éducatif ainsi que dans le monde du travail ; en témoignent l'ouverture d'écoles ou de cursus anglophones ou encore l'apparition de signalétique en anglais dans les rues par exemple.

Il parait nécessaire pour les Libanais d'être au minimum bilingues ou trilingues ; la diaspora de 15 millions de personnes joue un rôle important en ce sens. D'autant que le plurilinguisme apparaît comme un enrichissement pouvant lutter contre les stéréotypes, en travaillant également sur les représentations des langues. À ce titre, l'État libanais souhaite protéger le français, en signant notamment des accords de coopérations avec la France, afin également de pouvoir bénéficier de financements de formations de formateurs, d'écoles publiques francophones entre autres.

Le Liban est considéré comme étant le seul pays francophone de la région Moyen-Orient ; il est membre de l'Organisation Internationale de la Francophonie, et également membre fondateur de la Ligue Arabe ${ }^{9}$. Mais qu'en est-il réellement de la francophonie libanaise lorsque l'on voit se développer l'usage de la langue anglaise au sein de ce territoire?

\section{Les pratiques linguistiques au Liban : quel français développer et promouvoir ?}

Le contexte socioculturel et identitaire libanais n'est pas simple. Actuellement l'arabe, le français et l'anglais se côtoient dans ce pays de plus de quatre millions d'habitants.

\subsection{Le contexte libanais complexe : du bilinguisme au trilinguisme}

L'arabe est la langue officielle véhiculaire. L'arabe est l'unique langue officielle de l'État libanais. En effet, au sortir de l'indépendance le français n'a pas été choisi comme langue coofficielle au côté de l'arabe. Le français, surtout présent depuis le mandat en 1920, fut la langue mandataire dont le statut n'a jamais été défini après l'indépendance en 1943. Il est à noter que cette langue n'est pas forcément connotée négativement, car l'ancienne puissance mandataire a permis l'indépendance du Liban. Celle-ci est d'ailleurs reconnue langue d'enseignement au même titre que l'anglais (notamment pour les sciences).

L'anglais apparaît plus récemment, il est notamment institutionnalisé dans le secteur éducatif en 1997. D'après les données de notre enquête, cette langue est associée au commerce et au monde des affaires. L'apparition de l'anglais concurrence la langue française, jusqu'alors majoritaire avec l'arabe dans les processus d'apprentissage, car la langue anglaise prend progressivement de plus en plus de place au Liban, tant dans le système éducatif qu'en situations professionnelles.

Au Liban, l'arabe, langue officielle, n'est pas la seule langue utilisée à l'école. En effet, deux langues étrangères sont utilisées pour l'enseignement des matières scientifiques (les mathématiques et les sciences): le français ou l'anglais. Le système éducatif libanais est bilingue (arabe-français ou arabe-anglais). Les principales langues d'enseignement utilisées durant le primaire sont le français (à 68\%) et l'anglais (à 32\%). Dans l'enseignement secondaire, les pourcentages sont inversés. Durant le cycle supérieur, le français occupe 55\% de

\footnotetext{
${ }^{9}$ La Ligue arabe est une organisation d'États indépendants constituée en 1945 par l'Égypte, la Syrie, l'Irak, la Transjordanie, le Liban, l'Arabie Saoudite, le Yémen en vue de promouvoir leur coopération. Entre 1953 et 1993 , quatorze États et l'Organisation de Libération de la Palestine (OLP) y ont adhéré. L'Égypte, suspendue en 1979, a réintégré l'organisation en 1989.
} 
l'enseignement, l'anglais et l'arabe se partagent alors le reste. Comme nous l'avons déjà mentionné, 600000 élèves sur 900000 sont scolarisés dans des écoles francophones au Liban (tant en secteur privé ou qu'en secteur public) mais leur nombre diminue (de dix points en dix ans) selon les chiffres du Centre de Recherche et de Développement Pédagogique (CRDP) du ministère de l'Éducation. Si le français reste majoritaire dans l'enseignement public, en revanche, le secteur privé voit se développer de nombreuses écoles anglophones. Beaucoup d'élèves scolarisés en français adoptent l'anglais pour leurs études universitaires, essentiellement pour augmenter leurs chances d'emploi et leur capacité à être trilingue. Pourtant de nouveaux établissements francophones ouvrent leurs portes: les lycées privés francophones ont de longues listes d'attentes et les effectifs des universités francophones sont en augmentation ces dernières années.

Plusieurs profils de personnes au Liban peuvent être identifiés quant à l'usage des langues : des Libanais arabophones qui peuvent être bilingues, trilingues, des Libanais qui vont préférer travailler et utiliser le français en famille tout en parlant l'arabe (c'est-à-dire utiliser le français dans le cadre d'une socialisation). D'autres profils peuvent exister, car rien n'est homogène (il existe au Liban une communauté arménienne).

Les francophones aisés (chrétiens, chiites notamment) forment un groupe non chiffrable à cause des problèmes de recensement. Ils possèdent une influence non négligeable sur la scène nationale libanaise : dans les médias, certains ont des responsabilités étatiques (telle que responsable de radio publique francophone) et politiques (en tant que députés ou ministres). Cette minorité francophone et francophile construit un environnement francophone au Liban et refuse de se fondre « dans la masse », en conservant ses spécificités.

C'est ce groupe d'acteurs qui permet, par exemple, le dynamisme de la presse écrite libanaise francophone. Même si l'arabe est la langue fédératrice et officielle du Liban, le français conserve encore un rôle prépondérant dans les médias, et ce, grâce à une certaine "élite médiatique » qui continue de produire des journaux et des diffusions radiophoniques en français. La presse francophone n'est pas numéro 1 au Liban, puisqu'elle se place derrière la presse arabophone, mais elle demeure toutefois devant la presse anglophone, qui peine à se développer. Cependant sa place n'est pas négligeable. Même si son auditoire peut paraître restreint par rapport à la presse en général, la presse francophone possède l'image d'une presse de qualité. De nombreux titres existent en français au Liban, comme l'illustre la photo cidessous (cf. Figure 1). Ils vont du quotidien (l'Orient le Jour, Al balad), à la presse plus spécialisée (le Commerce du Levant), en passant par la presse de loisirs (comme Mondanité ou encore Femme magazine, premier mensuel féminin au Liban, et Déco magazine, un trimestriel existant depuis 2000).

Le français n'apparaît pas comme une langue de «commerce et de business »; c'est plutôt à l'anglais qu'est réservée cette image. Il apparaît donc intéressant de voir qu'un magazine spécialisé (d'une centaine de pages) traitant des questions commerciales et économiques au Moyen-Orient en français existe : Le Commerce du Levant. Ce mensuel fut crée en 1929; il est tiré à 7000 exemplaires par mois et il sort en kiosque à Paris. Quelquefois, une publication en arabe est possible, notamment lorsqu'un numéro spécial a connu de très bonnes ventes. Dans ce cas, il peut alors être traduit et vendu en arabe. Le français étant la langue de publication, il est alors nécessaire d'être francophone pour travailler au sein de la rédaction de ce magazine. Cependant, la rédactrice en chef, souligne lors de notre entretien que : « il est de plus en plus difficile de trouver des bons journalistes francophones, car la maîtrise de la langue n'est pas totalement parfaite, même si pourtant cela est nécessaire pour un journaliste ». 


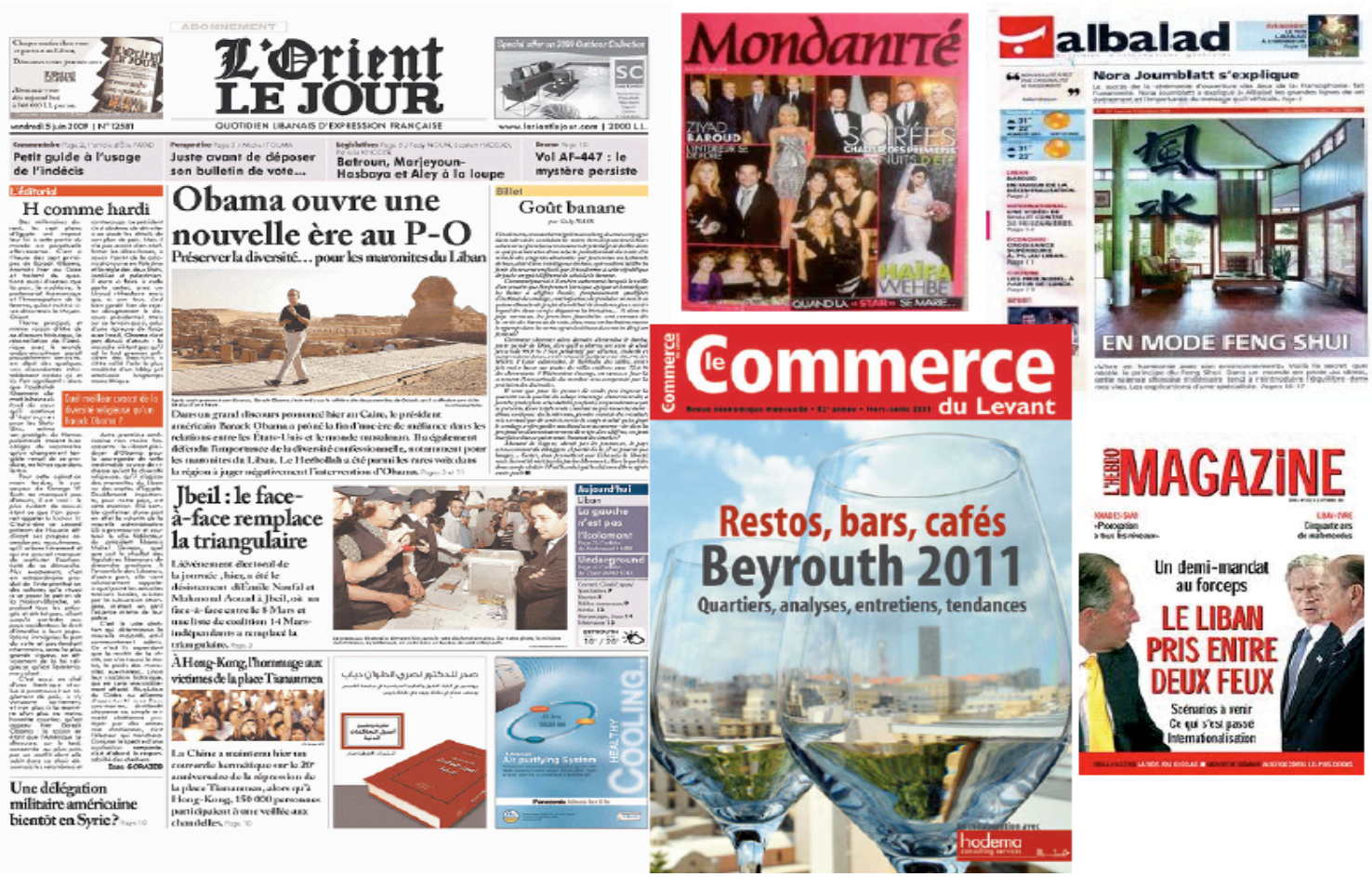

Figure 1 : Premières pages de différents journaux et magazines de la presse écrite francophone libanaise, 2011, MUNOZ Ludivynn

Sur les ondes, il existe également une radio publique francophone : Radio Liban en français. Elle n'est pas la seule radio francophone présente au Liban, il existe aussi par exemple Radio Nostalgie, une radio privée qui reprend le même principe que celle qui existe en France.

\subsection{Quelle didactique du français développer au Liban?}

Plusieurs points de vue s'opposent au sujet de la didactique du français à développer. Certains acteurs rencontrés pensent que le français est une langue d'usage quotidien que les Libanais maîtrisent et ils souhaitent donc développer le Français Langue Seconde (désormais FLS). D'autres en revanche, pensent que le français n'est pas forcément bien maîtrisé et ils prônent le Français Langue Étrangère (désormais FLE). Aucun choix réel n'est effectué, les deux sont donc développés. Il existe par conséquent un certain flou institutionnel, d'autant que les contextes et les profils sont multiples.

A ce titre, l'ambassade de France rencontre des problèmes avec ses interlocuteurs et collaborateurs au sujet du statut de la langue française. La principale question est de savoir quel français doit être développé et proposé aux Libanais. S'agit-il du français langue première, du FLS ou du FLE? Au Liban, suivant le type de population visé, ces trois statuts peuvent coexister. Par exemple, l'Université Saint Esprit de Kaslik (USEK) assume le FLE, alors que l'Université Saint Joseph (USJ) reconnaît seulement le FLS (dans cette université, la maîtrise du français doit donc être parfaite). Il existe des écoles qui se sont engagées auprès du ministère pour établir un état des lieux concernant les modalités de l'enseignement du français dans les écoles primaires, en vue de futures améliorations. 
En même temps que les moyens de l'ambassade de France diminuent (Renaud, 2010), les demandes augmentent, car les problèmes de formations sont importants ; même si le nombre de locuteurs francophones ne diminue pas, voire augmente, en revanche, la qualité de la maîtrise de la langue s'affaiblit (comme le soulignaient les attachés de coopérations de l'ambassade de France et des rédacteurs en chefs de journaux francophones). L'ambassade de France au Liban précise que le FLE peut être plus favorisé, même si elle continue de promouvoir le FLS dans ses coopérations avec le ministère de l'éducation libanaise.

L'ambassade de France a un rôle essentiel pour la présence et le maintien du français au Liban. Au Liban, elle est une des plus importantes du réseau diplomatique français, car il s'agit d'une ambassade à mission élargie. Il a été possible de rencontrer des personnes travaillant au sein du Service de Coopération à l'Action Culturelle (SCAC), un service indépendant des autres, que proposent l'ambassade comme le consulat, l'Agence Française de Développement. L'ambassade de France ne bataille pas réellement contre l'anglais (comme ont pu me l'indiquer les personnes rencontrées lors d'entretiens) et se place plutôt dans une politique d'action complémentaire, en prônant un "Liban trilingue » (arabe/français/anglais) où les personnes peuvent accéder au français grâce à l'anglais et vice versa. L'attachée de coopération pour le français prend en exemple le lycée français situé en face de l'ambassade qui va envoyer des élèves dans divers établissements francophones ou anglophones. Un tiers des élèves partent pour l'Université Saint Joseph (USJ), un autre tiers pour l'étranger, et le dernier part pour l'American University of Beirut (AUB). Selon une des personnes rencontrées le français « est instrumentalisé, il peut être un instrument de discrimination car il est très associé à l'élitisme ». L'ambassade de France s'évertue, surtout, à stabiliser la langue française, notamment en convaincant certaines universités francophones qui souhaitent passer à l'anglais (comme l'Université Saint Esprit de Kaslik - USEK - par exemple ; cf. Figure 2), de persister dans le français.

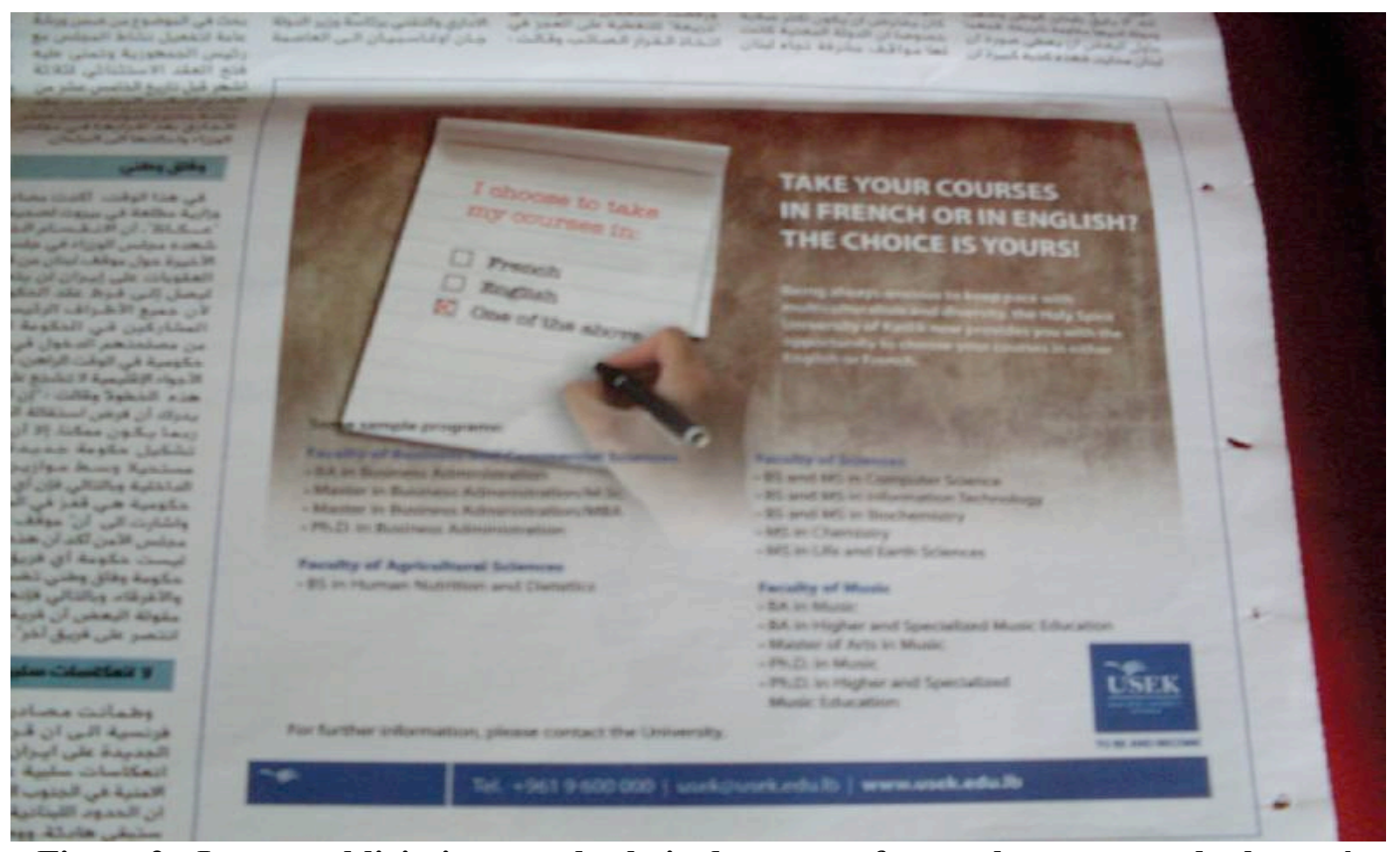

Figure 2 : Image publicitaire pour le choix des cursus francophones ou anglophones à l'Université Saint-Esprit de Kaslik dans l'édition arabophone du journal Al Balad du samedi 12 juin 2010, MUNOZ Ludivynn 
Les politiques d'action en matière de protection de la langue française peuvent se résumer en trois points : consolider et améliorer la qualité de l'enseignement de et en français au niveau préscolaire, scolaire et universitaire grâce à la certification (aide du CECR); soutenir la formation pédagogique pour de futurs enseignants qui prennent la relève en renforçant leurs compétences linguistiques, puisqu'on constate que même si le nombre de francophones augmente avec la scolarisation, le niveau de langue baisse; développer l'environnement culturel francophone (par exemple, proposer des manifestations culturelles francophones comme l'organisation du salon du livre francophone tous les deux ans, tenu fin octobre).

A ce titre, le 2 juillet 2009 a été distribuée en présence de Baya Hariri, alors ministre de l'éducation, une « mallette francophonie » à 80 écoles publiques, 90 bibliothèques publiques et Centre de Lecture et d'Animation Culturelle (CLAC), des bureaux pédagogiques privés, des médiathèques des Centres Culturels Français (CCF). Cette mallette fut financée par 10 ambassades francophones, dont la Suisse, la Bulgarie, le Canada, le Maroc. Il s'agit d'un support d'enseignement ludique du français avec des bandes dessinées, des romans, des cédéroms, des fichiers pour jouer en classe et un jeu sur la francophonie. Le but de cette mallette est de faire adopter aux formateurs une perspective "actionnelle » d'apprentissage (liée à une pédagogie qui se veut " active »). Par exemple, il peut être demandé aux élèves à qui est présentée la carte d'une ville marocaine, de découvrir les points communs existants entre les villes libanaises et marocaines, puis que chacun puisse réaliser le plan de son quartier; ou toujours selon cette approche constructiviste en éducation, la confection d'une brochure touristique peut être demandée après l'étude de celle proposée par la Suisse. On tente alors d'ouvrir plus les élèves libanais à la Francophonie et sa diversité qu'à la langue française en particulier. Surtout que le Liban est représenté comme l'emblème de la Francophonie au Moyen-Orient.

L'Université Saint Joseph (USJ) de Beyrouth, université francophone privée, réputée dans tout le Moyen-Orient (des Égyptiens francophones viennent suivre des masters par exemple), astreint ses futurs étudiants à passer un test d'aptitude en français (cf. Figure 3). Cette université promeut le FLS, car pour réussir leurs études les étudiants se doivent de maîtriser le français, puisque la majeure partie des enseignements et des examens se réalise en langue française. Pour s'inscrire dans tout cursus initial (excepté celui des lettres orientales), les étudiants doivent réussir le test d'aptitude, même ceux qui souhaitent étudier la médecine ou l'ingénierie comme le montre l'affiche qui suit. Les médecins formés dans cette université pourront choisir de travailler dans des pays francophones à l'étranger (France, Canada, Belgique par exemple) ou à l'Hôtel Dieu de France à Beyrouth (centre hospitalier universitaire) ou encore ouvrir un cabinet dans un quartier francophone de la capitale.

L'université organise ces tests pour que les étudiants suivent au mieux les enseignements proposés ; de plus elle souhaite "maintenir la qualité de la langue française dans ses enseignements » et "veut permettre aux bacheliers de combler des déficits de pratique du français ».

En effet, comme le soulignaient plusieurs journalistes et enseignants interviewés, la bonne maîtrise du français a tendance à diminuer. De plus, il y a des problèmes dans la formation des enseignants qui ne maîtrisent pas parfaitement le français et qui pourtant doivent prodiguer des enseignements dans cette langue. 


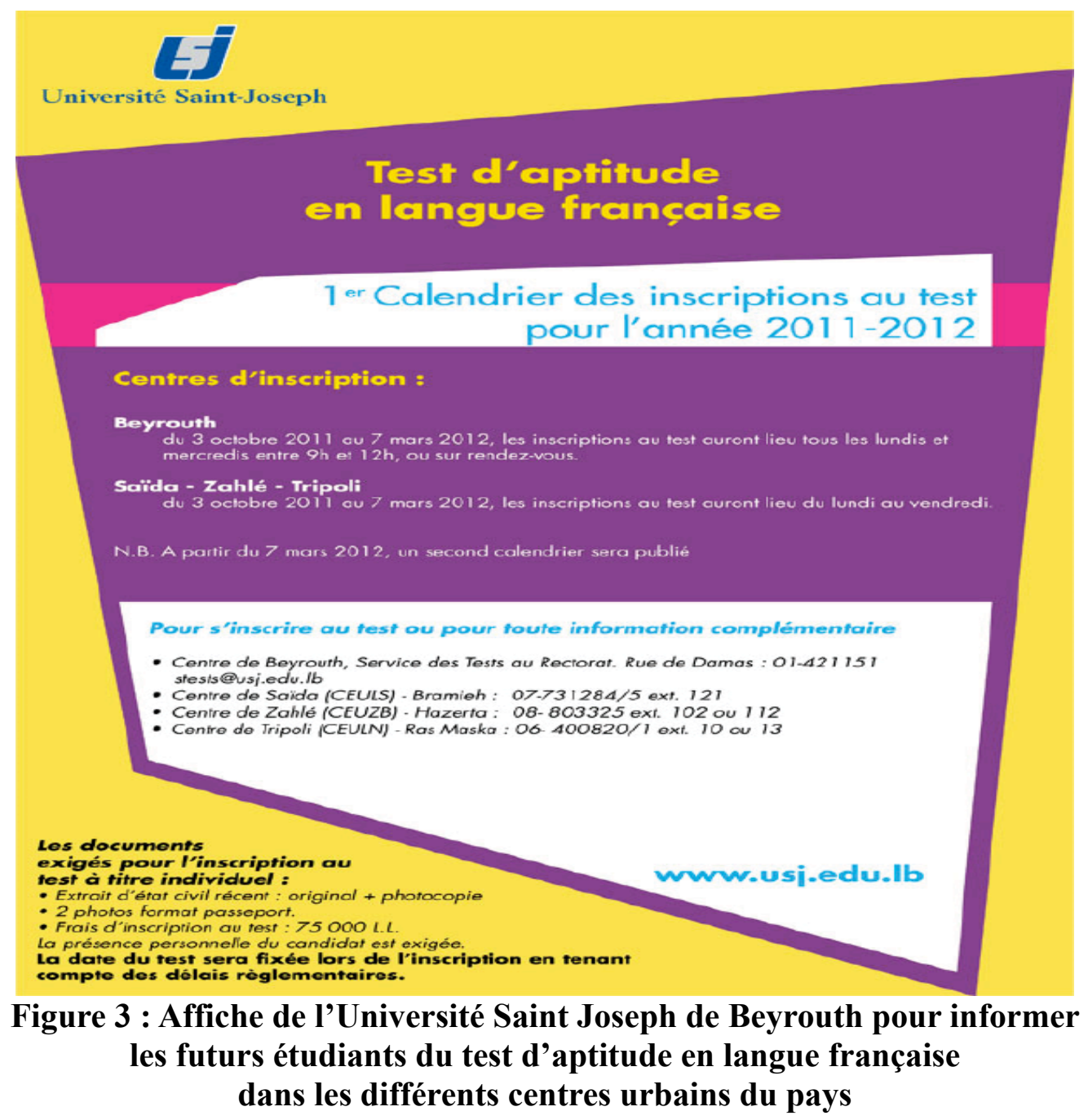

\section{Conclusion}

Le recours à la géopolitique apparaît nécessaire pour mieux cerner les contextes, car elle étudie les différents groupes d'acteurs présents sur un territoire qui peuvent s'affronter pour le contrôle de ce territoire. L'accès aux données n'est pas aisé compte tenu des tensions existantes dans ce pays. Cependant, il est possible de préciser que sur le territoire libanais de nombreux profils de personnes évoluant dans des contextes différents sont à prendre en compte. Par exemple, certains francophones chrétiens vont être scolarisés dans une école chrétienne, tandis que des francophones musulmans chiites, arabophones vont apprendre le français dans les écoles publiques. Ainsi, selon leur profil, les personnes ne vont pas se répartir sur les mêmes territoires et dans les mêmes établissements. Il apparaît difficile d'établir un choix quant au type de français à développer sur le territoire, car il existe comme notre enquête a pu le déterminer des profils forts différents. Ceci nous invite à poser deux ordres de questionnement linguistique, l'un didactique et l'autre géopolitique.

Notre premier questionnement est d'ordre didactique. Ne faudrait-il pas dès lors préconiser de développer plusieurs didactiques du français adaptées aux différents profils de stratégie linguistique vis-à-vis de l'usage du français, plus ou moins mobilisé dans le cadre d'une socialisation primaire en plus que dans celui d'une socialisation secondaire, mis au jour dans notre étude ? Même si une telle approche n'apparaît pas aisée à mettre en œuvre en raison des coûts potentiellement imposés (notamment par la formation et l'adaptation des formateurs à de 
tels profils), il pourrait être intéressant de constituer des expérimentations didactiques. Mais de telles expérimentations nécessitent, outre le fait de parfaire nos premiers résultats d'enquête, une collaboration avec des didacticiens de l'apprentissage des langues en vue de constituer de telles adaptations didactiques. Une telle collaboration reste à mener.

Concernant le questionnement de la géopolitique des langues, les francophones vont-ils se tourner vers l'anglais ou conserver leurs spécificités? Une étude plus approfondie de la population francophone serait souhaitable pour savoir ce que va réaliser à l'avenir cette population pour maintenir ses acquis. L'État libanais doit-il constituer des choix pour le français? Le pacte linguistique qu'il vient de signer (23 octobre 2010) avec l'Organisation Internationale de la Francophonie (OIF) va peut-être clarifier les choix pour les années à venir. Le pacte repose sur une démarche volontaire du pays signataire. Il se compose d'un plan d'action et d'un calendrier avec un suivi et une évaluation régulière (les pactes diffèrent d'un pays à l'autre pour répondre aux besoins de chaque État). Trois opérateurs de l'OIF sont mobilisés pour la bonne réalisation des plans d'actions des pactes linguistiques : l'Agence Universitaire de la Francophonie (AUF), TV5 Monde, l'Association de Internationale des Maires Francophones (AIMF). Le but du pacte linguistique libanais est «d'entretenir une dynamique favorable au français pour conserver et renforcer le caractère trilingue du Liban ». La place de l'anglais n'est plus contestable. Le plan d'action de ce pacte comporte trois volets : l'enseignement, la culture et l'environnement francophone. Qu'en sera-t-il de sa mise en œuvre?

\section{Références bibliographiques}

Breton, R. (2003). Atlas des langues du monde. Paris : Autrement.

Breton, R. (2006). Horizons et frontières de l'esprit, comprendre le multiculturalisme. Marseille : Le mot et le reste.

Berger, P. et Luckmann, T. (1966). La construction sociale de la réalité. Paris : Armand Colin.

De Montbrial, T. et Jansen, S. (2007). Géographie et Politique. Bruxelles : Bruylant.

Dubar, C. (1991). La socialisation. Construction des identités sociales et professionnelles. Paris : Armand Colin.

Faour, S., Velut, S. et Verdeil, E. (2007). Atlas du Liban. Paris : Ifpo/CNRS Liban.

Fitouri, C. (1983). Biculturalisme, bilinguisme et éducation. Paris : Delachaux et Niestlé.

Hagège, C. (2008). Langue et puissance. Géopolitique, Revue internationale de Géopolitique, 100, 27-33.

Giblin, B. (2007). Géopolitique de la langue française. Hérodote, Revue de géographie et de géopolitique, 126, 3-8.

Giblin, B. (2010). Bruxelles : le nœud gordien de la Belgique. Dans D. Papin (dir.), Cinquante fiches pour comprendre la géopolitique (p. 208-211). Paris : Bréal.

Khoury, G. (2006). Une tutelle coloniale, Le mandat français en Syrie et au Liban. Paris: Belin.

Lacoste, Y. (2003). De la géopolitique aux paysages, Dictionnaire de géographie. Paris : Armand Colin.

Lacoste, Y. (2009). Géopolitique, la longue histoire d'aujourd'hui. Paris : Larousse.

Plasseraud, Y. (2005). Atlas des minorités en Europe. Paris : Autrement.

Renaud, F. (2010). Les diplomates : derrière la façade des ambassades de France. Paris: Nouveau Monde éditions.

Rjeoutski, V. (2007). La langue française en Russie au siècle des Lumières : éléments pour une histoire sociale. Dans U. Haskins-Gonthier et A. Sandrier (dir.), Multilinguisme et multiculturalité dans l'Europe des Lumières (p. 101-126). Paris : Honore Champion. 